\title{
Correction: Ablation of dynamin-related protein 1 promotes diabetes-induced synaptic injury in the hippocampus
}

Gyeongah Park, Jong Youl Lee, Hye Min Han, Hyeong Seok An, Zhen Jin, Eun Ae Jeong, Kyung Eun Kim, Hyun Joo Shin, Jaewoong Lee, Dawon Kang, Hyun Joon Kim, Yong Chul Bae and Gu Seob Roh (D

Correction to: Cell Death and Disease

https://doi.org/10.1038/s41419-021-03723-7

published online 5 May 2021

The original version of this article unfortunately contained two mistakes. In the Materials and methods and the Acknowledgement section it should read "Dr. Jeanho Yun (Dong-A University, Busan, South Korea)". The authors apologize for the mistake. The original article has been corrected.

Published online: 01 June 2021 